\title{
Epidemiologia e Estatística: Integrando Ensino, Pesquisa, Serviço e Comunidade
}

\author{
Epidemiology and Statistics: Integrating \\ Teaching, Research, Services, and the \\ Community
}

Estelita Pereira Lima

\author{
PALAVRAS-CHAVE \\ - Educação Médica. \\ - Ensino \\ - Pesquisa. \\ - Aprendizagem. \\ - Bioestatística. \\ - Epidemiologia.
}

Recebido em: 12/08/2008

Reencaminhado em: 28/03/2009;

Reencaminhado em: 19/08/2009

Aprovado em: 17/09/2009

\section{RESUMO}

O novo modelo de saúde brasileiro exige médicos dotados de visão social abrangente e capacitados para prestar à comunidade cuidados permanentes e resolutivos, bem como profissionais críticos diante da tomada de decisões. Este relato descreve uma experiência estratégica de ensino de epidemiologia e bioestatística no curso de Medicina, cuja construção do processo foi centrada no educando, que foi o agente dos conhecimentos adquiridos, por meio da produção de informações e da pesquisa na comunidade ou nos serviços de saúde. Esta estratégia resultou na integração e interdisciplinaridade acadêmica, contribuindo para a compreensão de assuntos clínicos considerando a abordagem epidemiológica e estatística. Os acadêmicos despertaram para a importância da produção científica e da construção do currículo profissional ainda na fase acadêmica, sendo capazes de identificar problemas locais e direcionar as intervenções, e reconhecer que isto representa um dos objetivos mais importantes da pesquisa em saúde. Os resultados indicam que a epidemiologia e a bioestatística podem ser trabalhadas no curso de Medicina com o mesmo sucesso de outras disciplinas da clínica, desde que seja favorecida a aprendizagem significativa dos acadêmicos.

\section{ABSTRACT}

The new Brazilian health model requires physicians with a comprehensive social vision that are trained to provide the community with high-quality care on an on-going basis and are capable of making critical decisions. The current article describes a strategic teaching experiment in epidemiology and biostatistics during undergraduate medical training. The experiment focused on the students themselves as protagonists in acquiring knowledge through the production of information and research in both the community and health services. The strategy resulted in academic integration and interdisciplinarity, contributing to the grasp of clinical topics through an epidemiological and statistical approach. Students realized the importance of scientific output and the development of a professional resume while still in their undergraduate training and proved capable of identifying local problems and managing interventions, acknowledging the latter as a key health research objective. The findings indicate that epidemiology and biostatistics can be taught as successfully as other clinical courses during undergraduate medical training, as long as meaningful learning by students is encouraged. 


\section{INTRODUÇÃO}

Os tradicionais currículos dos cursos de Medicina têm se mostrado insuficientes para transmitir aos acadêmicos a relevância das ciências básicas para a prática clínica ${ }^{1}$. Integrando o conjunto dessas ciências, encontra-se a estatística, ministrada como bioestatística nos cursos da área da saúde. Entretanto, a inserção da bioestatística nestes cursos tem sido objeto de discussão entre professores e estudantes, em especial porque, comumente, grande parte dos estudantes a vê como uma disciplina complexa e cuja aplicabilidade é de difícil compreensão. Tal concepção pode ter contribuído para o desinteresse demonstrado por alguns acadêmicos e até para a aversão ao estudo de bioestatística, dificultando o processo ensino-aprendizagem e, em último caso, contribuindo para a evasão dos cursos. Desta forma, é preciso empregar estratégias de ensino que mostrem a importância da bioestatística para o curso de Medicina e para a prática clínica.

O emprego destas estratégias tem sido um desafio para as escolas médicas, que têm encontrado uma solução ao unirem a bioestatística com outras disciplinas, como, por exemplo, a epidemiologia e a medicina baseada em evidências.

A bioestatística auxilia na produção do novo modelo de médico, exigido pela medicina moderna. Entre outros pressupostos, devem se formar profissionais capacitados para a leitura crítica dos fenômenos que ocorrem na população relacionados ao processo saúde-doença, bem como selecionar e adotar com segurança novas tecnologias divulgadas rotineiramente em seu meio, tais como a formulação de novos medicamentos, novas técnicas cirúrgicas, identificação de grupos ou de fatores de risco para uma doença e alterações nos parâmetros de diagnósticos.

Ainda assim, a demonstração da aplicabilidade da bioestatística exige criatividade, intenso planejamento das atividades e aplicação de ferramentas tecnológicas modernas que auxiliem na exploração dos conhecimentos desta área, contextualizados com a saúde, que despertem nos acadêmicos a vontade de aprender. Freire ${ }^{2}$ defendia que "ensinar não é transferir conhecimento, mas criar as possibilidades para a sua própria produção ou a sua construção" (p.52). Portanto, o educador deve adotar posturas que transformem o estudante em agente ativo de sua aprendizagem.

Percebe-se, então, que é preciso adotar métodos pedagógicos progressistas, em que o educando aprenda a fazer fazendo ${ }^{3}$. No ensino médico, é necessário ir além. $\mathrm{O}$ acadêmico deve aprender a ser um médico humanista, a trabalhar em equipe, a refletir e a criticar, a solucionar problemas e a conhecer a si mesmo.

Partindo destes pressupostos, desenhou-se uma estratégia capaz de promover a aprendizagem significativa entre os aca- dêmicos, ou seja, aprendizagem com significado, com entendimento, pressupondo a aquisição de conhecimento "útil"4. É importante enfatizar que prover as condições para essa aprendizagem não é o mesmo que ensinar. Cardoso Júnior ${ }^{5}$ afirma que:

Deve-se motivar e fomentar a curiosidade do aprendiz que, por seus meios e de maneira ativa, assistido por facilitadores, deverá galgar as competências esperadas. Por sua vez, o significado de competência não se restringe à aquisição de um considerável corpo de informações, mas se refere, também, ao desenvolvimento de um conjunto de capacidades expressas no profissionalismo, nas habilidades de comunicação, no trabalho colaborativo e, especialmente, na efetividade da resolução dos problemas cotidianos. (p.21)

A estratégia aqui descrita envolve os acadêmicos em atividades científicas nas quais é preciso empregar os conhecimentos adquiridos ou construídos durante o módulo Assistência Básica à Saúde (ABS) — epidemiologia e bioestatística. Trata-se de uma disciplina obrigatória, com carga horária de 72 horas/aula, ministrada durante o terceiro semestre do curso de Medicina do Cariri, Universidade Federal do Ceará (UFC), sediado no município de Barbalha.

As atividades programadas exigem dos acadêmicos o desenvolvimento de uma pesquisa epidemiológica em equipe, inserção na comunidade ou no serviço de saúde e um tratamento estatístico, desde o planejamento do estudo à análise dos dados. Esse modelo dá oportunidade aos acadêmicos de pensar, discutir, produzir juntos e conhecer a realidade populacional e os serviços de saúde locais.

Este trabalho relata uma experiência estratégica de ensino de epidemiologia e bioestatística em medicina, em que a construção de todo o processo foi centrada no educando e na qual ele foi o agente dos conhecimentos adquiridos.

\section{MÉTODO}

A carga horária do módulo ABS - epidemiologia e bioestatística é distribuída em 18 dias, durante um semestre. $\mathrm{O}$ módulo é teórico-prático, com aulas semanais com a duração de quatro horas, sendo duas horas destinadas à aula teórica, e as outras duas, à aula prática ministrada no laboratório de informática. As aulas práticas têm por objetivo desenvolver nos acadêmicos habilidades operacionais em um programa estatístico, mediante resolução de exercícios, criação e análise de bancos de dados. Para este fim, a UFC adotou o software Epi-Info ${ }^{6}$. 
Trata-se de um software desenvolvido pelo Center for Diseases Control and Prevention (CDC) para a manipulação de dados epidemiológicos e que representa uma ferramenta indispensável para alcançar os objetivos de aprendizagem do módulo. Com o auxílio deste, os acadêmicos podem analisar bancos de dados complexos de forma simples e precisa, o que manualmente se tornaria inviável ou inseguro.

\section{IMPLEMENTAÇÃO DA ESTRATÉGIA}

Esta experiência foi iniciada em 2002 e ainda é empregada. A cada semestre, no primeiro contato com os acadêmicos, sonda-se a percepção e as expectativas dos acadêmicos em relação ao módulo. Nesse momento, também se explica a relevância da inserção da bioestatística no currículo, as vantagens de estudá-la junto com a epidemiologia e sua importância para a prática médica. Neste caso, todos os temas de bioestatística propostos para a disciplina são explorados como ferramenta para a compreensão e avaliação do processo saúde-doença na coletividade, bem como para o planejamento e análise de desenhos epidemiológicos, visando contribuir para a prevenção e controle dos agravos à saúde em nível local. Todos os exercícios e outras atividades realizadas são elaborados com base em casos clínicos ou em estudos epidemiológicos.

Ainda no primeiro dia de aula, são apresentados aos acadêmicos os conteúdos a serem estudados com seus respectivos objetivos, bem como o método de trabalho e de avaliação adotado, que consiste em avaliações formativas e somativas.

A avaliação formativa é aplicada, principalmente, para avaliar o desempenho e as habilidades dos acadêmicos a cada aula de informática, por meio da participação e resolução das atividades, relacionadas a condições de saúde-doença, com enfoque clínico-epidemiológico.

Segundo Sá, a avaliação formativa é utilizada para monitorar o progresso da aprendizagem e tem como objetivo promover um feedback contínuo entre aluno e facilitador. Já a avaliação somativa "avalia o processo para atingir objetivos de aprendizagem ao final da instrução"7(p.191). De acordo com o autor, para alcançar sucesso nesta escolha, um dos meios é utilizar múltiplos métodos de avaliação do progresso dos acadêmicos e as diferentes formas de expressar sua compreensão, desempenho e atitudes.

A avaliação somativa é aplicada duas vezes durante o módulo e tem como objetivo principal avaliar conhecimentos. A primeira ocorre, geralmente, na metade do semestre letivo e se trata de uma avaliação de conhecimentos teórico-práticos. As questões práticas são resolvidas no laboratório de informática, onde grupos de dois ou três acadêmicos analisam um banco de dados, criado por eles mesmos, com dados hipotéticos. $\mathrm{O}$ objetivo desta avaliação é estimular os acadêmicos a dominar o programa e a testar seus conhecimentos estatísticos antes de analisar dados verdadeiros, o que só ocorre no final do semestre. A segunda avaliação consiste na realização de uma pesquisa epidemiológica, que é apresentada e discutida em sala de aula.

Com a realização da pesquisa espera-se aplicar os conhecimentos estatísticos adquiridos durante as aulas, desde o delineamento até a análise de desenhos epidemiológicos e a aproximação entre os acadêmicos e a comunidade, visando torná-los conhecedores das condições sociais, ambientais e de morbimortalidade da população local. Outro propósito é ativar a capacidade intelectual e científica dos acadêmicos, desenvolver neles o senso crítico e inseri-los no Sistema Único de Saúde, utilizando-o como um campo prático de aprendizado, segundo orientam as novas Diretrizes Curriculares.

As pesquisas são realizadas em grupos de, no máximo, quatro acadêmicos, visando promover a integração de conhecimentos e capacidades, exercitando a postura de cada um numa equipe multidisciplinar e preparando-os para atender às exigências do atual mercado de trabalho.

Os temas das pesquisas são escolhidos pelos próprios acadêmicos. O educador os orienta a selecionar temas relevantes para a comunidade local ou para os serviços de saúde e que sejam interessantes para o grupo. Em nenhum momento os alunos devem ser pressionados a pesquisar sobre um tema que não seja de interesse para o grupo, pois a execução da pesquisa deve ser uma produção prazerosa e enriquecedora intelectualmente. Este pensamento é compartilhado com Breilh ${ }^{8}$ ao mencionar que:

O ponto de vista de um investigador consiste na perspectiva social a partir da qual focaliza seu objeto de trabalho, perspectiva esta que determina suas escolhas e rejeições, sua maneira de interrogar a realidade, o tipo de vínculo que estabelece com o processo que estuda, isto é, as mediações formais e práticas que se intercalam entre ele (como sujeito) e seu objeto. (p.44)

Quando a escolha de determinado tema envolve aspectos clínicos além dos epidemiológicos, médicos especialistas e educadores são convidados a integrar o grupo de pesquisa como co-orientador, promovendo a interdisciplinaridade acadêmica.

Durante as aulas, os acadêmicos conhecem os conteúdos de epidemiologia e bioestatística, e, ao mesmo tempo, as ferramentas necessárias a todas as etapas da pesquisa. Procurase variar os recursos audiovisuais com a utilização de quadro 
branco, projetor de mídia e microcomputador, de acordo com a adequação a cada conteúdo. Os assuntos são divididos de forma a facilitar a aplicação direta à pesquisa, desde a fase de planejamento até a análise dos dados. No decorrer do semestre, há permanente orientação dos trabalhos em todas as fases da pesquisa e em todos os aspectos metodológicos, técnicos e éticos.

O educador é responsável por abrir o caminho para os acadêmicos pesquisadores entrarem em campo, ou seja, encaminhá-los aos serviços de saúde, munidos de cartas de apresentação que relatam o teor da pesquisa. Se esta for realizada com dados secundários, após autorização dos gestores destes serviços, os dados são coletados antes de qualquer consolidação, isto é, a partir dos prontuários, fichas hospitalares e outros registros semelhantes. Dá-se preferência à busca de dados neste nível, devido à presença de informações mais detalhadas sobre o agravo em questão. A exploração dos bancos de dados do Sistema Nacional de Informação ocorre de forma complementar para orientar a discussão dos achados e a avaliação das condições de saúde local, com base na comparação dos indicadores de saúde nacionais.

Após a conclusão dos estudos realizados nos serviços de saúde, é obrigatória a entrega de uma cópia do trabalho no local. Muitos gestores já fazem esta exigência antes de autorizar a realização da pesquisa no serviço.

Quando a investigação envolve diretamente seres humanos, os discentes são orientados a enviar seus projetos ao Comitê de Ética em Pesquisa da UFC e a como abordar as pessoas durante a coleta de dados. Os projetos de pesquisas que precisam cumprir esta exigência são elaborados nos primeiros meses do semestre, para que o parecer do Comitê seja emitido em tempo hábil para a realização das pesquisas até o final do semestre.

A obtenção de dados primários na comunidade ocorre mediante a aplicação de questionários, formulários e entrevistas. Nenhuma pesquisa é desenhada com exigência de diagnóstico clínico ou laboratorial, já que não há recurso financeiro destinado a estas atividades.

Ao finalizarem a coleta de dados secundários ou primários, os acadêmicos criam seus bancos de dados e empregam o tratamento estatístico adequado a cada tipo de estudo. Finalizada esta etapa, os trabalhos são redigidos segundo as normas científicas e entregues para apreciação do professor. Após as correções, os acadêmicos preparam uma apresentação com o emprego de equipamentos didáticos audiovisuais.

A finalidade principal da apresentação é habilitar os acadêmicos a participar de eventos científicos. Assim, o educador recomenda que se respeitem os critérios de postura, comuni- cação científica e tempo. Estimula-se também que os colegas participem como espectadores ativos, a fim de criar um ambiente semelhante ao dos encontros científicos.

\section{RESULTADOS}

Em seis anos, 240 estudantes (20 por semestre) participaram da estratégia de ensino, sem nenhuma evasão escolar registrada e apenas uma reprovação. No mesmo período, foi registrada uma evasão do curso de Medicina e uma reprovação em outra disciplina.

A produção científica na faculdade e a participação dos acadêmicos em eventos científicos regionais e nacionais aumentaram substancialmente. A cada semestre foram produzidas, em média, dez pesquisas epidemiológicas sobre diferentes temas. $\mathrm{O}$ estudo dos aspectos clínicos foi estimulado também, pois era necessário conhecer o assunto para realizar as investigações.

Por meio das pesquisas, muitos agravos à saúde foram caracterizados no município de Barbalha e em outros que o circundam. Tornou-se conhecido o perfil epidemiológico de algumas doenças endêmicas na região, como leishmaniose, hanseníase, diabetes e doenças sexualmente transmissíveis. Foram investigadas as condições de saúde de trabalhadores de várias categorias, como agricultores, agentes sanitaristas e professores. Investigou-se também a ocorrência de acidentes automobilísticos e possíveis determinantes, acidentes domiciliares, condições de sexualidade e fatores associados à gravidez na adolescência. Outros trabalhos foram realizados nas áreas de mortalidade infantil, saúde do idoso, nutrição infantil na escola, saúde ambiental, neoplasias, desvios posturais, hemodiálise, transplante renal, sistema de informação em saúde, alcoolismo, tabagismo, saúde mental e aleitamento materno.

Além destes, analisou-se uma série histórica de oito anos dos acidentes causados por animais peçonhentos em Barbalha e realizou-se um inquérito sobre ato médico em escolas de saúde da região. Dos trabalhos realizados, três ganharam prêmios em encontros científicos; um artigo foi publicado na íntegra; nove foram publicados sob a forma de anais em periódicos de circulação nacional; e sete sob a forma de anais em revista estadual. Quanto aos outros, pelo menos 50\% foram publicados sob a forma de anais em congressos regionais e nacionais.

O objetivo de capacitar os estudantes para a elaboração de projetos de pesquisa foi alcançado, considerando-se que cerca de 30 deles foram beneficiados com bolsas de iniciação científica e de extensão. $\mathrm{O}$ maior ganho foi a capacidade que adquiriram para identificar os problemas locais e direcionar as 
intervenções, e reconhecer que isto representa um dos objetivos mais importantes da pesquisa em saúde.

Considera-se que as metas da estratégia foram alcançadas, com superação de expectativas. O estudo da epidemiologia e da bioestatística contribuiu para a interdisciplinaridade acadêmica e para a compreensão de assuntos clínicos considerando outras abordagens. Os acadêmicos despertaram para a importância da produção científica no curso de Medicina e da construção do currículo profissional ainda na fase acadêmica.

Algumas situações reveladas orientaram a elaboração de projetos de extensão que beneficiaram a comunidade regional por cerca de três anos. Entre eles se destacam os projetos: "Prevenção de acidentes automobilísticos"; "Prevenção de acidentes domiciliares causados por produtos químicos"; "Educação em saúde para o trabalhador rural" e "Nutrição saudável na infância". Outras ações de extensão continuam em curso, tendo como alvo a saúde dos grupos de idosos, materno-infantil, diabéticos, comunidades de assentamento, entre outros. Estas ações são coordenadas por professores de variadas especialidades e envolvem grupos de acadêmicos de vários semestres, garantido a manutenção e a renovação das atividades.

Embora não seja possível comparar esta estratégia de ensino com outras aplicadas anteriormente, já que foi implantada logo na primeira turma do curso de Medicina do Cariri, os resultados confirmam que a epidemiologia e a bioestatística podem ser trabalhadas em medicina com o mesmo sucesso de outras disciplinas da área de saúde. Para isto, devem ser adotadas metodologias didáticas capazes de estimular os acadêmicos a utilizá-las como ferramentas importantes na prática clínica, para a compreensão e resolução dos agravos à saúde.

\section{REFERENNCIAS}

1. Penaforte JC. Medicina como ciência, medicina como arte: um conflito educacional. Rev Educ Poucas Palavras. 2006;4:13.
2. Freire P. Pedagogia da autonomia: saberes necessários à prática educativa. São Paulo: Paz e Terra; 1996.

3. Libâneo JC. Democratização da escola pública: a pedagogia crítico-social dos conteúdos. São Paulo: Loyola; 1990.

4. Tomaz JBC. Em busca da aprendizagem significativa: o uso de mapas conceituais em um currículo PBL. Rev Educ Poucas Palavras. 2006;4:17.

5. Cardoso Júnior A. Acadêmicos liderando acadêmicos: a facilitação, entre pares, em um curso de graduação. Rev Educ Poucas Palavras. 2006;4:21.

6. Epi Info [computer program] Version 6. Atlanta, GA: Centers for Disease Control and Prevention; 1994.

7. Sá HLC. Avaliação dos acadêmicos. In: Mamede S, Penaforte J, orgs. Aprendizagem baseada em problemas: anatomia de uma nova abordagem educacional. São Paulo: Hucitec; 2001.

8. Breilh J. Epidemiologia: economia, política e saúde. São Paulo: Ed. Unesp; 1991.

\section{CONFLITO DE INTERESSES}

Declarou não haver.

\section{ENDEREÇO PARA CORRESPONDÊNCIA}

Estelita Pereira Lima

Rua Ivani Feitosa, 123

Tiradentes - Juazeiro do Norte

CEP 63031-140 - CE

E-mail: estelitaplima@hotmail.com 